Distaler Radius

\section{Bedeutung der Ulna- styloidfraktur für das Outcome nach distaler Radiusfraktur}

Almedghio S et al. Effects of Ulnar Styloid Fractures on Unstable Distal Radius Fracture Outcomes: A Systematic Review of Comparative Studies. J Wrist Surg 2018; 7: $172-181$

Bei distalen Radiusfrakturen gehören Frakturen des Processus styloideus ulnae zu den häufigsten Begleitverletzungen. Eine Behandlung dieser distalen Ulnafraktur gilt vielen Autoren nur dann als indiziert, wenn ansonsten die Stabilität des distalen Radioulnargelenks beeinträchtigt wäre.

Andere Experten meinen dagegen, dass die nicht behandelte Fraktur langfristig zu chronischen Handgelenkschmerzen bis hin zu arthrotischen Veränderungen führen kann. Inwieweit solche nicht versorgten distalen Ulnafrakturen die Ergebnisse im Verlauf beeinflussen, haben Mediziner aus Großbritannien untersucht. Sami Almedghio und seine Kollegen haben dazu in den einschlägigen medizinischen Literaturdatenbanken nach bis Januar 2014 veröffentlichten Arbeiten gesucht, in denen instabile distale Radiusfrakturen operativ behandelt worden waren und für begleitende Frakturen des Processus styloideus ulnae, wenn vorhanden, keine spezifische Therapie erfolgte. In die Auswertung gingen 6 Studien mit insgesamt $796 \mathrm{~Pa}$ tienten ein (806 distale Radiusfrakturen), darunter lag

- bei 444 Frakturen eine begleitende Fraktur des Processus styloideus ulnae vor (55\%, Gruppe 1), und

- in 362 Fällen eine isolierte distale Radiusfraktur (45\%; Gruppe 2).

Die Wissenschaftler prüften nun, ob die Originalarbeiten zwischen diesen beiden Gruppen zum Zeitpunkt der letzten Nachuntersuchung Unterschiede bei funktionellen Ergebnissen (im Wesentlichen anhand des DASH-Werts [Disabilities of the Arm, Shoulder, and Hand]), Bewegungsumfang, Kraft und Schmerzen auf einer visuellen Analogskala (VAS) von 0 (keine Schmerzen) bis 10 (stärkste vorstellbare Schmerzen) gefunden hatten. Bei den Studien handelte es sich um eine randomisierte klinische Studie, eine Fall-Kontroll-Studie und 4 vergleichende Beobachtungsstudien (3 prospektive, eine retrospektiv). Die durchschnittliche Nachbeobachtungszeit lag zwischen 15 und 24 Monaten.

- In 3 Studien fanden die Originalautoren beim DASH-Wert keine signifikanten Unterschiede zwischen den Gruppen, 2 Studien dagegen berichteten über schlechtere funktionelle Ergebnisse bei begleitender Ulnafraktur.

- Zwei Studien ergaben signifikant stärkere Schmerzen an (wenn auch mit einem wohl klinisch nicht relevanten Unterschied von 0,7 VAS-Punkten) bzw. häufigere Schmerzen bei Patienten der Gruppe 1.

- Keine Studie zeigte einen signifikant stärkeren Kraftverlust in Gruppe 1.

- Nur eine Studie fand beim Bewegungsumfang (Flexion/Extension, Pronation/Supination, Radial-/Ulnarabduktion) eine eingeschränkte Flexion in Gruppe 1 (54 vs. $59^{\circ}$ in Gruppe 2).

\section{FAZIT}

Auch ihre Auswertung kann keine abschließende Aussage dazu treffen, ob bei einer distalen Radiusfraktur eine begleitende Fraktur des Processus styloideus ulnae zwingend versorgt werden muss, so die Autoren. Die eine verfügbare randomisierte Studie ( $n=91)$ immerhin hatte bis auf geringfügig stärkere Schmerzen keinen relevanten Unterschied bei nicht versorgter Ulnafraktur (im Vergleich zur isolierten Radiusfraktur) gezeigt. Größere randomisierte Studien müssten die Frage aber weiter untersuchen.

Dr. Elke Ruchalla, Bad Dürrheim

\section{Kommentar}

Die Autoren der vorliegenden Arbeit haben sich eines Themas angenommen, das seit einiger Zeit kontrovers diskutiert wird: Hat die Fraktur des Processus sty- loideus ulnae (PSU) als Begleitverletzung der distalen Radiusfraktur einen Einfluss auf das Ergebnis? Ist sie in der basisnahen Variante gar ein Indikator für Instabilität des distalen Radioulnargelenkes (DRUG)? Die Aussagen dazu sind in der Literatur nicht einheitlich: Auf der einen Seite stehen die Verfechter einer operativen Stabilisierung mit der Intention, eine Instabilität des DRUG zu vermeiden, die bekanntlich zu schlechten funktionellen Ergebnissen führt. Dem gegenüber steht die Auffassung anderer, die grundsätzlich keine Osteosynthese des PSU vornehmen, gestützt von Daten aus der AOCID Prospective ORIF Distal Radius-Studiengruppe. Um nun herauszufinden, wessen Weg am ehesten der richtige ist, wurde hier ein systematisches Review durchgeführt. Für dieses Thema sicher nicht der schlechteste Ansatz, denn Veröffentlichungen dazu sind zahlreich vorhanden - 369 konnten die Autoren in einer aufwendigen Suche finden. Jedoch mussten anhand der von ihnen selbst gewählten Qualitätskriterien 351 Artikel wieder ausgeschlossen werden. Das zeigt eines: Die Güte der Veröffentlichungen über dieses Thema ist eher mäßig, will sagen: die Aussagekraft niedrig. Genau dies wollten die Autoren mit ihrem Review der verbliebenen 6 Publikationen und 796 Patienten verbessern, indem sie ihre Gütekriterien zum Einschluss streng ansetzten.

Bei näherem Hinsehen ist der Anspruch nicht ganz erfüllt worden. Es findet sich nur eine einzige randomisierte kontrollierte Studie darunter. Auch sind die Ergebnisse der sechs Patientenkollektive nicht vergleichbar, da keine einheitlichen Messmethoden zur Beurteilung von Funktion und subjektiver Einschätzung verwendet wurden. Insgesamt ist die Qualität der Arbeiten nicht befriedigend. Es wurden nur Publikationen in englischer Sprache verwendet. In zwei Studien wurden die Ausschlusskriterien nicht benannt, lediglich in zwei anderen wurde eine ausreichende Kollektivgröße statistisch gesichert. Es wurde in keiner Arbeit zwischen Niedrigenergietraumata bei älteren und Hochenergietraumata bei jüngeren unterschieden, obwohl bekannt ist, dass letztere mit einer höheren Rate an Begleitverletzungen gerade des 
ulnaren Kompartiments und schlechterer Funktion korreliert sind. Teils wurde die Altersgrenze der Patienten zum Studienausschluss bei unter 18 Jahre, teils bei unter 40 Jahren angesetzt. Es wurden nur Studien eingeschlossen, in denen keine Stabilisierung des PSU vorgenommen wurde. Das könnte zu einer Verzerrung der Ergebnisse führen, wenn man voraussetzt, dass die PSU-Frakturen mit Instabilität des DRUG in höherem Maße operativ versorgt werden.

Wichtige Aspekte der Versorgung der distalen Radiusfrakturen, die bekanntlich erheblichen Einfluss auf die gemessenen Parameter wie Griffkraft, DASH, Bewegungsumfang haben, sind nicht berücksichtigt worden:

- Es wurde nicht nach Behandlungsmethoden differenziert. Zur Auswertung kamen winkelstabile Plattenosteosynthesen, Fixateur externe, Kirschnerdraht-Fixation, geschlossene Reposition und Ruhigstellung im Gips.

- Die Qualität der Reposition - ein wesentlicher Faktor für die Stabilität des DRUG - wurde nicht berücksichtigt.
Man liest also einen Review, dessen Aussage - das Vorliegen einer Fraktur des PSU habe keinen Einfluss auf den Outcome einer (wie auch immer behandelten) Radiusfraktur - auf wackligen Beinen steht. Mulders und Mitarbeiter haben in einer in diesem Jahr aufgelegten Metaanalyse mit 12 Publikationen dieselbe Aussage getroffen, aufgrund der vergleichbaren Methodik ebenfalls nicht sehr belastbar. Die Frage, wie und ob der PSU als Begleitverletzung der distalen Radiusfraktur relevant ist, bleibt letztendlich unbeantwortet.

\section{Autorinnen/Autoren}

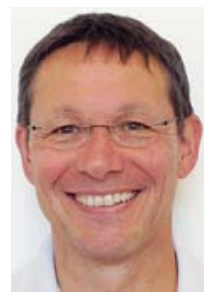

Dr. Peter Laier, Leitender Oberarzt, Facharzt für Chirurgie, Facharzt für Orthopädie und Unfallchirurgie, Facharzt für spezielle Unfallchirurgie, Facharzt für Handchirurgie, Unfall-, Hand- und Orthopädische Chirurgie, Städtisches Klinikum Karlsruhe 